Journal of Applied Pharmaceutical Science Vol. 7 (01), pp. 204-208, January, 2017

Available online at http://www.japsonline.com

DOI: $10.7324 /$ JAPS.2017.70130

ISSN 2231-3354 (cC) BY-NC-SA

\title{
Physicochemical Characterization of Physical Mixture and Solid Dispersion of Diclofenac Potassium with Mannitol
}

\author{
Yong K. Han ${ }^{\mathrm{a}}$, Sonia N. Faudone ${ }^{\mathrm{b}}$, Gustavo Zitto ${ }^{\mathrm{b}}$, Silvina L. Bonafede ${ }^{\mathrm{a}}$, María A. Rosasco ${ }^{\mathrm{a}}$, Adriana I. Segall ${ }^{{ }^{*}}$ \\ ${ }^{a}$ Universidad de Buenos Aires, Facultad de Farmacia y Bioquímica, Cátedra de Calidad de Medicamentos, CONICET, Junín 956, (1113), Buenos Aires, \\ Argentina. ${ }^{\mathrm{b}}$ CEPROCOR Centro de Excelencia en Productos y Procesos de Córdoba, Álvarez de Arenales 230, X5004AAP, Córdoba, Argentina.
}

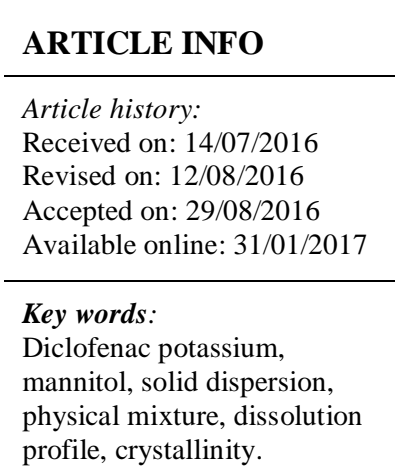

\begin{abstract}
Diclofenac potassium is an anti-inflammatory agent classified as a class II drug as per the biopharmaceutical classification system (BCS). The poor dissolution rate of water-insoluble drugs is still a major problem confronting the pharmaceutical industry. There are several techniques to enhance the dissolution of poorly soluble drugs. Methods available include salt formation, micronization and addition of solvent or surface active agents. The objective of the present work is to improve the dissolution profile of diclofenac potassium by formation of a physical mixture and a solid dispersion with mannitol. The solid dispersion was prepared by solvent method using ethanol/water. As diclofenac potassium melts with decomposition, the compatibility study with mannitol was done with the acid form by differential scanning calorimetry (DSC). The dissolution properties and physicochemical properties of diclofenac potassium:mannitol physical mixture and solid dispersion were investigated by Powder X-ray Diffraction (PXRD), Fourier Transform Infrared Spectroscopy (FTIR), Scanning Electron Microscopy (SEM) and dissolution test. This study shows that the dissolution rate of diclofenac potassium can be enhanced considerably by formulating it with mannitol, as a physical mixture or as a solid dispersion although crystallinity was maintained.
\end{abstract}

\section{INTRODUCTION}

Diclofenac is commercially present as sodium and potassium salt in tablets for oral administration and as diethylamine for topical application. While extensive literature is available for sodium salt little has been reported on the potassium salt (Fini et al., 2001). The solid dispersion technique for waterinsoluble drugs developed by Chiou and Reigelman (Chiou and Reigelman, 1971) provides an efficient method to improve the dissolution rate of a drug (Ford, 1986; Leuner and Dressman, 2000; Frizon et al., 2013). In solid dispersion systems, a drug may exist as an amorphous form in polymeric carriers, and this

\footnotetext{
* Corresponding Author

Adriana I Segall, Universidad de Buenos Aires, Facultad de Farmacia y Bioquímica, Cátedra de Calidad de Medicamentos, CONICET, Junín 956, (1113), Buenos Aires, Argentina.Email: asegall @ffyb.uba.ar
}

may result in improved solubilities and dissolution rates as compared with crystalline material. Several investigators have proposed the mechanisms for the enhancement of the dissolution rate of solid dispersions. Drugs molecularly dispersed in polymeric carriers may achieve the highest levels of particle size reduction and surface area enhancement, which result in improved dissolution rates (Craig, 2002). Furthermore, no energy is required to break up the crystal lattice of a drug during dissolution process (Taylor and Zografi, 1997), and drug solubility and wettability may be increased by surrounding hydrophilic carriers (Craig, 2002). The methods used to prepare solid dispersions include the melting method, the solvent method and the solvent wetting method (Leuner and Dressman, 2000; Yamashita et al., 2003). However, the melting method and the solvent method have some limitations. In the case of the melting method, incomplete miscibility between drug and carrier may occur due to the high 
viscosity of a polymeric carrier in the molten state and thermally unstable drugs can be degraded due to the requirement of relatively high preparation temperatures. In the case of the solvent method, since both drug and carrier must be dissolved completely in organic solvent, subtle alterations in the conditions used for solvent evaporation may lead to large changes in product performance (Leuner and Dressman, 2000; Moneghini et al. 1998). The solvent method also introduces residual solvent, which may bring up the environmental issues.

Mannitol was used to increase the aqueous solubility and dissolution of different drugs (Rajko et al., 2009; Zajc et al., Arias et al., 1995; 2005; Arias et al., 1998). Diclofenac potassium has been classified as a class II drug as per the biopharmaceutical classification system (BCS) (Chuasuwan et al., 2009).

The objective of the present work is to improve the dissolution profile of diclofenac potassium by formation of a physical mixture (PM) and a solid dispersion (SD) with mannitol. The solid dispersion was prepared by solvent method using ethanol/water.

As diclofenac potassium melts with decomposition, the compatibility study with mannitol was done with the acid form by differential scanning calorimetry (DSC). The dissolution properties and physicochemical properties of diclofenac potassium:mannitol physical mixture and solid dispersion were investigated by Powder X-ray Diffraction (PXRD), infrared spectrometry (FTIR), scanning electron microscopy (SEM), and dissolution test.

\section{MATERIALS AND METHODS}

\section{Materials}

Mannitol (purity $\geq 99.0 \%$ ) was received as a gift sample from Merck S.A. (Merck, Germany). Diclofenac potassium (purity $99.9 \%$ ) was purchased in Saporiti, Argentina. Methanol used was HPLC grade, Sintorgan (Buenos Aires, Argentina) and monobasic sodium phosphate AR Grade, J. T. Baker (Estado de Mexico, Mexico). Distilled water was passed through a $0.45 \mu \mathrm{m}$ membrane filter. All the solvents and reagents used were of analytical or HPLC reagent grade.

\section{Preparation of physical mixture and solid dispersion Physical Mixture}

A PM of $5 \mathrm{~g}$ of diclofenac potassium and mannitol (1:1) by weight was prepared by mixing with spatula in a mortar until a homogeneous mixture was obtained. The resulting mixture was sieved through a \# 80 sieve

\section{Solid dispersion prepared by solvent evaporation}

A mixture of $5 \mathrm{~g}$ of diclofenac potassium and mannitol (1:1) by weight respectively was wetted with water-ethanol (1:1 ratio) The solvent was evaporated under reduced pressure at $40{ }^{\circ} \mathrm{C}$, and the resulting residue was dried under vacuum for $3 \mathrm{~h}$, stored in a desiccator at least overnight, ground in a mortar, and passed through a \# 80 sieve.

\section{Diclofenac Acid (for DSC)}

The free acid was obtained by dissolving diclofenac potassium in water and lowering the $\mathrm{pH}$ using perchloric acid $0.01 \mathrm{~N}$ until $\mathrm{pH}: 3.0$. The precipitate was recovered by vacuum filtration. The precipitate was washed with distilled water until neutrality and then, dried at $40{ }^{\circ} \mathrm{C}$ under vacuum using a vacuum oven.

\section{Analysis of the active ingredient}

The liquid chromatographic method was carried out on a

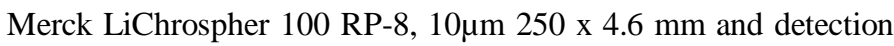
was performed at $254 \mathrm{~nm}$. The mobile phase consisted of $0.01 \mathrm{M}$ monobasic sodium phosphate buffer:methanol $(30: 70 \mathrm{v} / \mathrm{v}), \mathrm{pH}$ adjusted to 2.5 using phosphoric acid. The flow rate was $1 \mathrm{~mL} / \mathrm{min}$ and was maintained at room temperature. The chromatographic separation was obtained with retention time of $6.0 \mathrm{~min}$. The injection volume was $20 \mu \mathrm{L}$. The HPLC system consisted of a dual piston reciprocating Thermo Finnigan pump, a Rheodyne injector, a DAD Dionex Ultimate 3000 with operating software Chromeleon 6.8 was used during the study.

\section{Preparation of standard solution}

An accurately weighed quantity of $75 \mathrm{mg}$ of diclofenac potassium was dissolved in $25 \mathrm{~mL}$ of a mixture methanol:water (70:30) (diluent solution). One $\mathrm{mL}$ aliquot of the solution was transferred to a $10 \mathrm{~mL}$ volumetric flask, diluted to volume with diluent solution. The solutions were passed through a $0.45 \mu \mathrm{m}$ nylon membrane filter before injection ( $25 \mathrm{~mm}$ disposable filter; Cat. $\mathrm{N}^{\circ}$ R04SP02500 Osmonics Inc., Minnesota, USA).

\section{Sample Preparation}

Approximately $150 \mathrm{mg}$ of the SD or the PM were exactly weighed, placed into a $25 \mathrm{~mL}$ volumetric flask, taken to volume with diluent solution. One $\mathrm{mL}$ aliquot of the solution was transferred to a $10 \mathrm{~mL}$ volumetric flask, diluted to volume with diluent solution. The solutions were passed through a $0.22 \mu \mathrm{m}$ nylon membrane filter before injection $(25 \mathrm{~mm}$ disposable filter; Cat. $N^{\circ}$ R04SP02500 Osmonics Inc., Minnesota, USA).

\section{Differential scanning calorimetry (DSC)}

A differential scanning calorimeter (DSC 822, Mettler Toledo, Switzerland) was used for thermal analysis of drug and excipient. Individual samples (drug and excipient) as well as the physical mixture of the drug and the excipient were weighed directly in the pierced DSC aluminium pan and scanned in the temperature range of $30-350{ }^{\circ} \mathrm{C}$ under atmosphere of dry nitrogen. A heating rate of $10{ }^{\circ} \mathrm{C} / \mathrm{min}$ was used and the obtained thermograms were observed for any interaction. The DSC cell was calibrated with indium (m.p. $156.6^{\circ} \mathrm{C} ; \Delta H_{\text {fus }}=28.5 \mathrm{Jg}^{-1}$ ) and Zinc (m.p. $419.6{ }^{\circ} \mathrm{C}$ ) as standards. Empty aluminium pans were used as references. Samples with mass $1-2 \mathrm{mg}$ were employed, by duplicate. Data were treated with STARe software Ink. (Mettler Toledo, Switzerland). 


\section{Powder X-ray Diffraction (PXRD)}

PXRD were collected at RT using a D8 Advance X-ray diffractometer (Bruker AXS, Germany). The target was Coppertube $((\mathrm{Cu} \mathrm{K \alpha}$ radiation $\Theta=1.5418 \AA)$ and a post-diffraction graphite monochromator. The $\mathrm{X}$-ray generator was set at a voltage of $40 \mathrm{kV}$ and current of $40 \mathrm{~mA}$. Samples were subject to PXRD analysis in step mode with a step size of $0.05^{\circ} 2 \Theta$ and a step time of $3 \mathrm{~s}$ over an angular range of $2-50^{\circ} 2 \Theta$. The sample holder was rotated in a plane parallel to its surface at the speed of $30 \mathrm{rpm}$ during the measurements. Obtained diffractograms were analyzed with DIFFRACplus EVA diffraction software.

\section{Fourier Transform Infrared Spectroscopy (FTIR)}

Infrared spectra (4000-400 $\mathrm{cm}^{-1}$ ) were recorded on a Nicolet iS10 FT-IR spectrometer Thermo Scientific. Solid samples were placed over a diamond attenuated total reflectance (ATR) accessory (Smart iTR), without any grinding or $\mathrm{KBr}$. The spectra were collected with 32 scans, at $4 \mathrm{~cm}^{-1}$ resolution.

\section{Scanning electron microscopy}

Electron micrograph of potassium diclofenac, mannitol,

PM and SD were obtained using scanning electron microscope (SEM) Carl Zeiss Ligma at the Laboratorio de Microscopía y Análisis de Rayos X (LAMARX) of the National University of Córdoba. The samples were sputtered with gold using a Balzers model SDC 030. Micrographs with different magnifications were recorded to study the morphology of the samples.

\section{Dissolution rate studies}

Dissolution studies were performed using USP 38 (The United States Pharmacopeia, 2015) Apparatus 2 (Vankel, VK 7010). The pure drug, PM and SD were placed in capsules $\mathrm{N}^{\circ} 0$. The diclofenac potassium content in PM and SD was normalized by HPLC and the equivalent of $75 \mathrm{mg}$ was placed in each capsule. Tests were carried out with twelve units and were performed at 50 $\pm 1 \mathrm{rpm}$. Dissolution media was phosphate buffer $\mathrm{pH} 6.8$ at $37 \pm$ $0.5{ }^{\circ} \mathrm{C}$. Dissolution media volume was $900 \mathrm{ml}$. In all experiments, $10 \mathrm{ml}$ sample aliquots were withdrawn at 10, 15, 20, 30 and 45 min using syringes. All samples were filtered through blue ribbon filter paper. The amount dissolved was calculated by determining the absorbance of an appropriately diluted solution at $276 \mathrm{~nm}$.

\section{RESULTS AND DISCUSSION}

Diclofenac potassium melts with decomposition (Fini et al., 2001). Diclofenac potassium thermogram indicated that the starting material corresponds to the dehydrated form (Figure 1). The compatibility study with mannitol was done with the acid form by differential scanning calorimetry (DSC). The thermogram of diclofenac (acid form) exhibited a single, sharp, endothermic peak at temperature of $164.23{ }^{\circ} \mathrm{C}$, mannitol exhibited a melting point of $165.14{ }^{\circ} \mathrm{C}$ and the mixture exhibited a melting point of $165.03{ }^{\circ} \mathrm{C}$, as the addition of both peaks (Figure 2 ). The melting endotherm of diclofenac acid was well retained in the blend with mannitol. $\mathrm{T}$

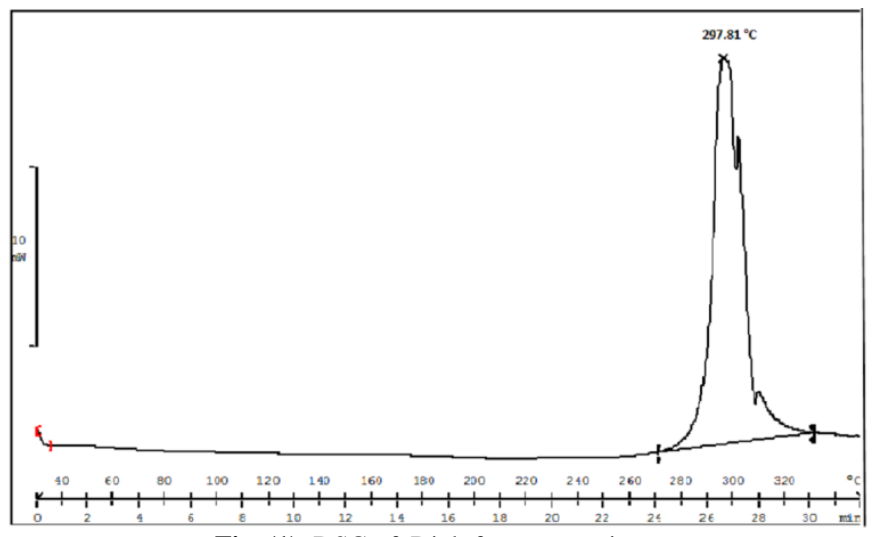

Fig. (1). DSC of Diclofenac potassium

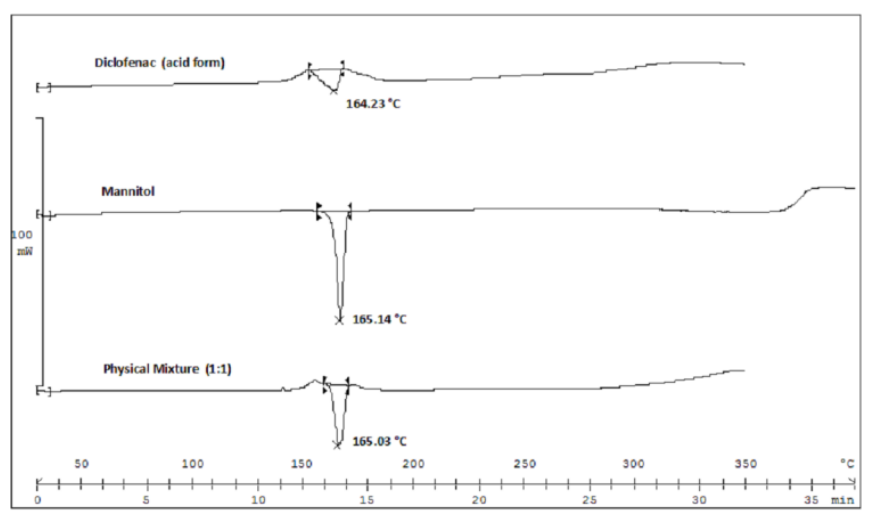

Fig. (2). DSC of Diclofenac (acid form), Mannitol, and Diclofenac (acid form):Mannitol 1:1 physical mixture.

The enthalpy value of PM is reduced to half the sum of the endotherms of diclofenac (acid form) and mannitol (Table I). The minor changes in the melting endotherm of the mixture could be attributed to the mixing of active and excipient, and not indicate potential incompatibility. Variations in the enthalpy values for the binary mixtures can be attributed to some heterogeneity in the small samples used for the experiments (3-4 mg) (Verma et al., 2005).

Table 1 Peak temperature and enthalpy values

\begin{tabular}{lccc}
\multicolumn{1}{c}{ Samples } & $\mathbf{T}_{\text {onset }}\left({ }^{\circ} \mathbf{C}\right)$ & $\mathbf{T}_{\text {peak }}\left({ }^{\circ} \mathbf{C}\right)$ & $\boldsymbol{\Delta H}\left(\mathbf{J g}^{-\mathbf{1}}\right)$ \\
\hline Diclofenac (acid form) & 153.85 & 164.23 & -87.85 \\
Mannitol & 163.51 & 165.14 & $-303,95$ \\
Physical mixture & 162.91 & 165.03 & -162.19 \\
\hline
\end{tabular}

The X-Ray diffractometry showed that pure diclofenac potassium, diclofenac potassium within PM and diclofenac potassium in SD were crystalline in nature (Figure 3). The pure diclofenac potassium showed the characteristics diffraction peaks at $2 \Theta$ of $14.4,15.3,16.5,21.7,25.3,26.1,27.0$, and $27.9^{\circ}$ etc., indicating the presence of crystalline diclofenac potassium. This powder pattern was concordant with the dehydrated form reported by Fini (Fini et al., 2005). 


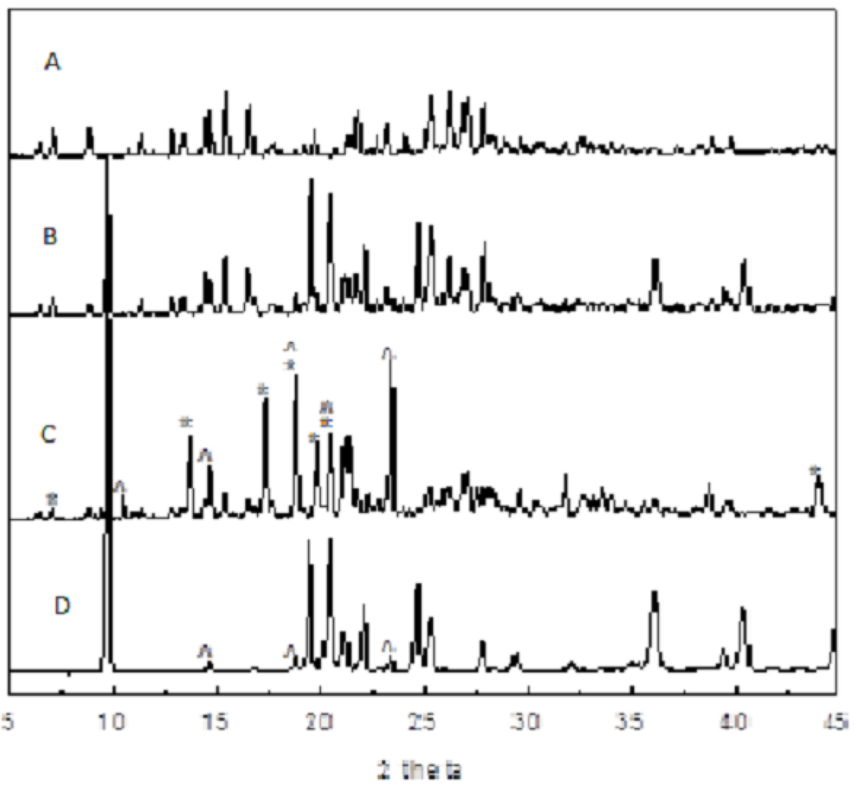

Fig. (3). Powder X-ray diffraction patterns of 1:1 Diclofenac potassiumMannitol test preparation. Key: A, raw Diclofenac K; B, physical mixture; C, solid dispersion, D, raw Mannitol. ^ Mannitol polymorph Beta. * Mannitol polymorph Alpha.

The pure mannitol showed the characteristics diffraction peaks at $2 \Theta$ of $9.7,19.5,20.4,22.1,24.7,25.3,36.1,40.4$ and $44.9^{\circ}$ etc., indicating the presence of crystalline mannitol, polymorphic form Delta with monoclinic $\left(\mathrm{P} 2_{1}\right)$ crystal structure as the mayor phase. The minor peaks observed at 14.6, 18.8 and $23.4^{\circ}$ $2 \Theta$ correspond to mannitol polymorph Beta (Orthorrombic, $\mathrm{P} 2{ }_{1} 2_{1} 2_{1}$ ) present as an impurity crystalline phase (Fronczek, 2003). The PM also showed crystallinity, due to the presence of crystalline diclofenac potassium and mannitol. Thus, the mere presence of mannitol in PM should not interfere with the characterization of coexisting diclofenac potassium. The X-ray diffraction pattern of PM was a superimposition of each component.

In case of the 1:1 ratio diclofenac potassium-mannitol $\mathrm{SD}$ also showed crystallinity, due to the presence of diclofenac potassium and mannitol. The X-ray diffraction pattern showed all reflections corresponding to crystalline diclofenac potassium, but distinct differences in another peak positions. As a result of reviewing the polymorphic forms of mannitol (Fronczek, 2003), it was possible to identify a mixture of polymorphs Beta (characteristic peaks at 10.5, 14.6, 18.8, 20.5, 21.2 and $23.4^{\circ} 2 \Theta$ ) and Alpha (characteristic peaks at 9.4, 13.7, 17.3, 18.7, 19.8, 20.4 and $43.9^{\circ} 2 \theta$ ) present in the SD sample.

This result implies that diclofenac potassium and mannitol are present in crystalline form in the 1:1 diclofenac potassium-mannitol PM and SD. Thus, there was no additional amorphization of the drug and which retained its original crystalline form, although mannitol polymorph Delta was transformed to a mixture of polymorphs Beta and Alpha in the SD.
The FTIR spectrum of pure diclofenac potassium showed a band at $3248 \mathrm{~cm}^{-1}$ due to the N-H stretching, a band at $2973 \mathrm{~cm}^{-1}$ due to the $\mathrm{C}-\mathrm{H}$ stretching, a band at $1575 \mathrm{~cm}^{-1}$ due to $\mathrm{C}=\mathrm{O}$ stretching, two typical bands at 1305 and $1449 \mathrm{~cm}^{-1}$ relative to the $\mathrm{C}-\mathrm{C}$ stretching, and at $745 \mathrm{~cm}^{-1}$ owing to $\mathrm{C}-\mathrm{Cl}$ stretching.

Mannitol shows a broad absorption band at $3229 \mathrm{~cm}^{-1}$ assigned to the hydroxyl groups. The spectra obtained from the 1:1 ratio PM presented band assignments at the same wavelength ranges depicting no interaction between the drug and mannitol The SD containing the 1:1 drug-mannitol showed that there was no shifting of the principal shoulders and the maximum of the drug with the use of mannitol. This confirms that there was no interaction between the drug and the mannitol used.

Scanning electron microscopy (SEM) (Figure 4) shows the aggregation of smaller crystals of pharmaceutically available diclofenac potassium (Fig 4 A) whereas mannitol has irregular sticks with a median volume diameter (Fig 4 B) and these two different crystal shapes can be easily identified in the physical mixture (Fig 4 C). It was observed that the solid dispersion sample obtained from methanol:water (70:30) contained crystalline particles with a smooth surface and a unique surface morphology different from diclofenac potassium and mannitol particles (Fig 4 D). This suggests that the two components were homogeneously distributed and a solid solution was formed which could be responsible for the increased dissolution rate of diclofenac potassium.
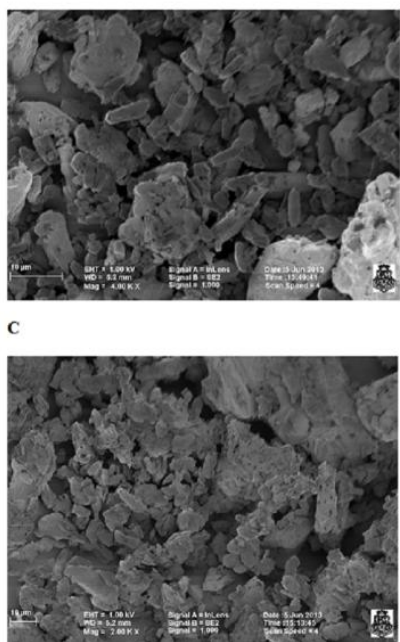
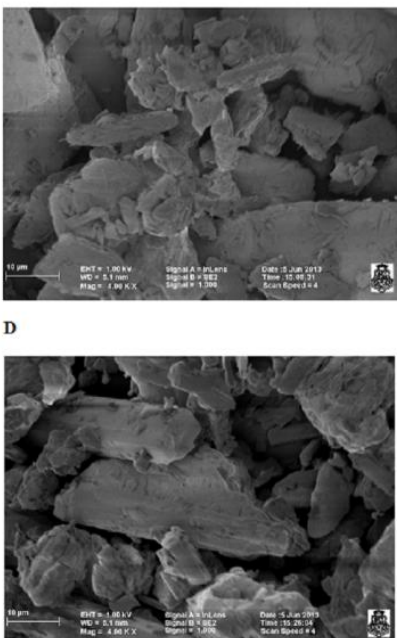

Fig. (4). Photographs (SEM of $10 \mu \mathrm{M}$ ) Key: A, Diclofenac potassium; B Mannitol; C, physical mixture; D, solid dispersion.

The effect of mannitol on the dissolution of diclofenac potassium was investigated. The pure drug dissolved approximately $60 \%$ in this condition. The dissolved amounts of diclofenac potassium from the 1:1 diclofenac potassium-mannitol PM increased 34\% compared with the pure drug. The diclofenac potassium:mannitol (1:1) SD increased the dissolved amount in $41 \%$ compared with the pure drug. Figure 5 shows the percent 
dissolved in function of the time, presenting the Standard deviation values at each time point of the dissolution testing.

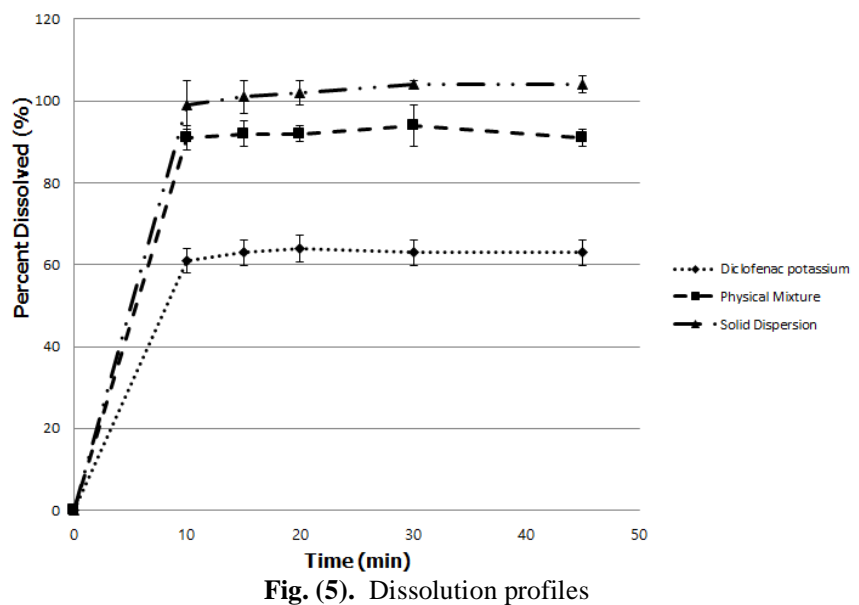

\section{CONCLUSIONS}

The solid dispersion of diclofenac potassium and mannitol was prepared using solvent evaporation. The physical mixture was prepared by thoroughly mixing appropriate amount of diclofenac potassium and mannitol in a mortar until a homogeneous mixture was obtained. The data obtained from DSC and FT-IR showed no significant drug-carrier interactions.

PXRD results indicated that diclofenac potassium is in crystalline form both in PM and SD, while mannitol ( $\delta$ polymorph) experienced a polymorphic transformation in the SD (polymorphs $\beta$ and $\alpha$ ).

This study shows that the dissolution rate of diclofenac potassium can be enhanced considerably by formulating it with mannitol, as a PM or as a SD in comparison with the pure drug. In both cases, crystallinity was maintained.

\section{ACKNOWLEDGEMENTS}

Financial support and sponsorship: This work was supported by grant 20020130100342BA to A. I. Segall from UBA and PIP No: 11420110100380 from CONICET. The authors also thank Merck S.A. (Argentina) for the donation of the mannitol.

Conflict of Interests: There are no conflicts of interest.

\section{REFERENCES}

Arias MJ, Ginés JM, Moyano JR, Pérez Martínez JI, Rabasco AM. Influence of the preparation method of solid dispersion on their dissolution rate: study of triamtirene-D-mannitol system, Int J Pharm, $1995 ; 123: 25-31$.

Arias MJ, Ginés JM, Moyano JR. Study by DSC and HSM of the oxazepam \pm PEG 6000 and oxazepam \pm D-mannitol systems: Application to the preparation of solid dispersions, Thermochim. Acta, $1998 ; 321: 33-41$.

Craig DQM. The mechanism of drug release from solid dispersion in water-soluble polymers, Int J Pharm, 2002; 231:131-144.

Chiou WL, Riegelman S. Pharmaceutical applications of solid dispersion systems, J Pharm Sci, 1971; 60:1281-1302.

Chuasuwan B, Binjesoh V, Polli JE, Zhang H, Amidon GL, Junginger HE, Midha KK, Shah VP, Stavchansky S, Dressman JB, Barends DM. Biowaiver monographs for immediate release solid oral dosage forms: Diclofenac sodium and diclofenac potassium, J Pharm Sci, 2009; 98(4):1206-19.

Fini A, Garuti M, Fazio G, Álvarez Fuentes J, Holgado MA. Diclofenac salts. I. Fractal and thermal analysis of soddium and potassium diclofenac salts. J Pharm Sci, 2001; 90:2019-2057.

Fini A, Fazio G, Rosetti F, Holgado MA, Iruín A, Álvarez Fuentes J. Diclofenac salts. III. Alkaline and Earth Alkaline Salts, J Pharm Sci, 2005; 94:2416-2431.

Frizon F, de Olivera EJ, Donaduzzi CM, Mitsui ML, Marchetti JM. Dissolution rate enhancement of loratadine in polyvinylpyrrolidone K-30 solid dispersions by solvent methods, Powder Technol, 2013; 235:532-539.

Ford JL. The current status of solid dispersions. Pharm Acta Helv, 1986; 61:69-88.

Fronczek FR, Kamel HN, Slaterry M. Three polymorphs $(\alpha, \beta$, and $\delta$ ) of D-mannitol at $100 \mathrm{~K}$. Acta Crystallogr, 2003; C59:0567-o570.

Leuner C, Dressman J. Improving drug solubility for oral delivery using solid dispersions, Eur J Pharm Biopharm, 2000; 50: 47-60.

Moneghini M, Carcano A, Zingone G, Perissutti B. Studies in dissolution enhancement of atenolol. Part I, Int J Pharm, 1998; 175:177183.

Rajko R, Nassab PR, Szabo-Revesz P. Self-modeling curve resolution method applied for the evaluation of dissolution testing data: A case study of meloxicam-mannitol binary systems, Talanta, 2009; 79:268 274.

Taylor LS, Zografi G. Spectroscopic characterization interactions between PVP and indomethacin in amorphous molecular dispersions. Pharm Res, 1997; 14:1691-1698.

The United States Pharmacopeia $38^{\text {th }}$ Ed. (Spanish version) U.S. Pharmacopeial Convention, Rockville, MD; 2015, 3341-3342.

Verma RK, Garg S. Selection of excipients for extended release formulations of glipizide through drug-excipient compatibility testing, J Pharm Biomed Anal, 2005; 38:633-644.

Yamashita K, Nakate T, Okimoto K, Ohike A, Tokunaga Y, Ibuki R, Higaki K, Kimura T. Establishment of new preparation method for solid dispersion formulation of tacrolimus, Int J Pharm, 2003; 26:7991.

Zajc N, Obreza A, Bele M, Srcic S. Physical properties and dissolution behaviour of nifedipine/mannitol solid dispersions prepared by hot melt method, Int J Pharm, 2005; 291:51-58.

\section{How to cite this article:}

Han YK, Faudone SN, Zitto G, Bonafede SL, Rosasco MA, Segall AI. Physicochemical Characterization of Physical Mixture and Solid Dispersion of Diclofenac Potassium with Mannitol. J App Pharm Sci, 2017; 7 (01): 204-208. 